\title{
Eine Einritzung auf der Pyramide des Mykerinos \\ Von der relativen (geschlechtlichen) Identität des Banalen und des Erhabenen
}

\section{Friedbert Aspetsberger}

Die menschliche Gattung (klassisch: der Mensch in seiner Menschheit) definiert sich vorgängig-fortzeugend, in der Produktion von Daten und in deren Erfahrung. Das zeigen die Ursprungs- oder Schöpfungsgeschichten der Mythen und Religionen, die jeweils geltenden Gesellschaftsformen, deren Naturbearbeitung, deren Kunst-, Technik- und Geschäftstraditionen. Die Gattung definiert sich darin - nochmals hölderlinisch klassisch: - als ein in sich selber Unterschiedenes (Heraklits Hen diaphéron heauto), als Unruhe und Arbeit, als ungewisses Postulat und insofern als Frage nach dessen Darstellbarkeit. Das heißt: die Gattung definiert sich in der Organisation bedürfnisgeleiteter Vorgänge zu allgemeinverbindlich gedachten "Bildern", "Bedeutungen", "Netzen" etc. Sie ist insofern fortzeugende Selbstinformation.

Die jeweils etablierten Leit-Bilder sind instabil: "es könnte ebensogut anders sein", dachte sich Gott, als er die Welt schuf, nach Robert Musils Roman Der Mann ohne Eigenschaften. Jedenfalls ist nicht gesagt, daß die "gegebenen" Normen (die bisherigen Data) und Bedeutungen nicht durch andere ersetzt sein könnten. Wie schwierig das aber ist, zeigt nicht nur der Roman Der Mann ohne Eigenschaften, es zeigen das auch gesellschaftliche Alternativformen wie z. B. konsequente terroristische Systeme, recente "primitive" Kulturen, die Arche Noah (mit ihrer Erhaltung der alten Arten) und die herrschende, bisher nicht seligmachende Organisation der Wirklichkeit im Kapitalismus, die als seligmachend beworben wird. Relationismus ist für alle Daten bzw. ihre "Bedeutungen" konstitutiv. Alle sind Daten des Historismus der Gattung, die sich ein multiversales Leben schon vorstellen kann, sich ihrer Relativität also bewußt ist. Ich gebe einige Beispiele zu den Spannungen von Daten und Bedeutungen, die ich willkürlich aus Gegenwart und Geschichte aufgreife. Ich verwende dabei die Begriffe "banal", "trivial" und "erhaben" unscharf abge- 
grenzt: im Sinn der gattungsgeschichtlichen (historistischen) Vermittlungsvorgänge wird als "banal" das Selbstverständliche und in der (bürgerlichen) communis opinio Nicht-Erwähnenswerte oder Verschwiegene des Lebensvollzuges verstanden, als "trivial" das Selbstverständliche und in der (bürgerlichen) communis opinio Erwähnenswerte, als "erhaben" die individuell (oder mit dem Anspruch von Individualität) verantwortete Interpretation des Banalen und Trivialen in seiner Historizität.

\section{Die Pyramide des Mykerinos}

Pyramiden sind eine bekannte Form und geschichtlich eine bekannte Beeutung; sie sind "der Himmel auf Erden". Zur Pyramide des Mykerinos gibt es mehrere Überlieferungen. Eine bezieht sich darauf, daß das Bauwerk steht und kleiner ist als die Cheops- und die Cheffren-Pyramide, neben denen sie steht, und erklärt das damit, daß Mykerinos aus sozialem Gewissen die Aufwendungen und Opfer für eine große Pyramide gescheut habe. Auf dieses Bild eines sozialen Mykerinos bezieht sich eine andere Überlieferung: Er hätte die Pyramide aus sozialem Gewissen nicht gebaut. Sie steht nach dieser Überlieferung, nicht von ihm für sein Bild von sich erbaut, sondern erbaut von Rhodopis, einer versklavten Hellenin, Nobelhure in Ägypten. Sie habe die Pyramide für ihr Bild von Mykerinos, der gegen die Ausbeutung wirkte, mit dem durch ihren ausgebeuteten Körper widerwillig verdienten Geld gestiftet. Die Pyramide überliefert also auch ein Bild von ihr. Die beiden - so wird man sagen müssen - die beiden Mykerinospyramiden dieser zwei Überlieferungen sprechen unterschiedliche weitere Bedeutungen an. Ich greife eine auf: Im Sinne der Gender Studies informiert der Bau der Rhodopis (im Format des Monuments Pyramide) z. B. über eine vorbestimmte Rolle, die Geschlechtsrolle der Frau, die Rhodopis, aufgrund ihrer Erfahrungen in dieser Rolle, nicht sein will und im Sinne ihrer Selbst-Formatierung als Pyramide auch nicht ist. Denn sie schafft ein sie überdauerndes Denkmal gegen jene Lebenszeit, die die meiste Zeit ihres vergehenden Körpers ausmachte; wir können ihrer pyramidal gedenken, wie sie selber es tat, also in ganz anderer Gestalt als sie als Hure war. "Information altert nicht" - sagt der InformatikDuden -, jedenfalls nur langsam bei Pyramiden und für Leser klassischer Real-Enzyklopädien; "nichts ist unsterblicher als ein Archiv", schreibt Günter Grass zur Subversivität wiederaufbereiteter Information in Zusam- 
menhang mit den StaSi-Berichten bei der Vereinigung der "deutschen" Staaten bzw. zweier Staaten mit Amts- und Umgangssprache Deutsch und zwischen 1949 und 1989 unterschiedlichen gesellschaftlichen Bedeutungen bestimmter Herkunft.

Rhodopis und wir erleben unsere Zeit, indem wir nicht sind, was wir in ihr sind und was die Zeit grundsätzlich ist: unsere Herkunft, eine Fortsetzung. Wir sind nicht nur Herkunft, z. B. das weibliche Geschlecht, das wir uns nicht aussuchen können. Robert Musils Figur Agathe sagt im Mann ohne Eigenschaften: "[...] es ist so schön, anders zu sein als man geboren wurde"; und sie will ihre bisher unfreiwillig dem Patriarchat geweihte Geschlechtlichkeit ändern (eine andere Frau, der Zwilling ihres Bruders werden, nicht die Tochter ihres Vaters sein). Es geht um Erfahrung der Data, um das, was in bestimmter Form als Wirklichkeit gemacht wird und so ist, aber auch anders sein könnte '(nämlich nicht-patriarchal). Die Pyramiden sind dauerhafte Speicher von Wünschen und Bedeutungen im Gefüge jener Grammatik, mit der sich das Menschengeschlecht darstellt. Aus dem Blick der Gott-Mensch-Pharaonen geben sie seit langem ("ewig") Nachricht von der Flüchtigkeit der menschlichen Leben und Tode und schützen ihr menschliches Datum im Innern eines gewaltigen Steinbergs mit glatter, "formschöner" Oberflächen-Verkleidung in einer geheimen Grabkammer (nicht ohne Grund: ein großzügiger Mumien-Import in die Vereinigten Staaten im 19. Jahrhundert führte dazu, daß der museal unverkäufliche Überschuß an Mumien schließlich zur Papierherstellung verwendet wurde; grundsätzlich blieb damit aber die Bedeutung der Mumifizierung, "Informationsträger" der Gattung zu sein, erhalten - auch als Zeugnis der neueren Informations-Kultur, die über Leichen geht, um die Gattung zu bestimmen).

Der Pyramiden-Bau korrigiert als Bedürfnis der Rhodopis die ihr aufgezwungene Wirklichkeit, ist in diesem Sinn Selbstbestimmung, d. h. Gegenwart als Verurteilung der Herkunft, hier des nicht selbstgewählten Geschlechts und seiner Umstände, z. B. der Versklavung. Er ist der Versuch einer eigenen Geschichte in der gattungsmäßig und gesellschaftlich-politisch vorbestimmten. Mit der Pyramide schreibt sich Rhodopis, geschlechtsrollenbestimmte Einzelne, in den "monumentalen Diskurs" der Gattung ein, in dem die Pyramiden stehen (J. Assmann).

Die Pyramide der Rhodopis ist demnach, unter vielem andern, als indi- 
viduelles und zugleich gattungsmäßiges Bekenntnis monumentaler Anspruch einer Unterdrückten gegen die Unterdrückung. Die Hure gehört der durch die Göttlichkeit des Pharao, der sozial dachte, definierten Gattung an. Insofern sind alle Informationen über das Menschengeschlecht gleich.

\section{Die erhabenen und die banalen (selbst die vulgären) Bedeutungen sind Eines}

Nicht jeder verdient so viel wie Rhodopis, um eine Pyramide zu bauen und sich damit in den monumentalen Diskurs der Gattung einzuschreiben. Bei geringerem Einkommen ritzt man seinen Namen in eine bestehende Pyramide ein, um seine ("ewige") Zugehörigkeit zum Menschheitsbild zu dokumentieren. Oder man liest statt der Einritzung eine klassische RealEnzyklopädie, wie ich das gemacht habe, oder kauft beworbene Informationen im "Sach"-Format, z. B. Off-Road-Geräte mit überbreiten Reifen. Die Realisierungen scheinen unterschiedlich, die Bedürfnisse sind gleich. Einritzungen an Pyramiden sind überliefert. Ebenso die Einritzung "O5", die an der Westwand des Stephansdomes von einer österreichischen Widerstandbewegung gegen Ende des Nazi-Regimes angebracht wurde, ein damals gleichermaßen andere Gegenwart fordernder Anspruch ( $\mathrm{O}$ und der fünfte Buchstabe des Alphabets signalisieren $\mathrm{OE}$ für Österreich, das damals abgeschafft war). Ebenso sind Einritzungen von Kirchen- und Schloßbesuchern in Fresken, Einritzungen an Aussichtspunkten, Einritzungen von damit als gegenwärtig erklärter (aber dem Wunsch-Partner vielleicht unerklärter) Liebe in Baumrinden, Einritzungen gegen Sanktionen aufgrund bestehenden Rechts in Gefängniswände, Einritzungen der Einsamkeit eines Geschlechts auf Closettwänden usw. überliefert. Mit der Geschlechtsnot hängt aber, wie erwähnt, auch das Monument der Rhodopis zusammen. Die erhabenen und die banalen, selbst die vulgären Bedeutungen sind als Kundgaben in Denk-Malen Eines (ein in sich Unterschiedenes). In Elias Canettis Roman Die Blendung flieht der Weltmeister der Wissenschaft, der Sinologe Professor Peter Kien, aus seiner monumentalen Bibliothek ins Closett, als er sein Geschlecht dem einer Haushälterin aussetzt; er verbrennt schließlich die monumentale Bibliothek (die er ins Closett auch nicht mitnehmen kann, um bei seinem Bild von sich zu bleiben) und verbrennt sich, weil er keinen Platz hat 
neben Therese bzw. weil der "männliche" Geist gegen die "weibliche" Geschlechtlichkeit nur schwer besteht: so nach dem JahrhundertwendeRepräsentanten Otto Weininger, den Canetti las. Das Verbrennen der Bibliothek - um es paradox zu sagen: - löscht die Bedeutung Mann/ Kien/Wissenschaftsweltmeister. Das Erhabene geht in der Blendung als Männlich-Erhabenes (im Rahmen der weiningerischen Mythologie unserer Geschlechterpolarität) im Banalen von Theresens Lesart der Wirklichkeit auf. Wenn auch nicht restlos: einerseits rettet sich Kien in das ideologisch hohe Bild der Selbstverbrennung, anderseits ist uns Weiningers "Einritzung" in die Gattungspyramide, sein Buch Geschlecht und Charakter (1903), durch mehr als vierzig Auflagen monumental, und ist uns Canettis Roman Die Blendung monumental als Werk eines Nobelpreisträgers. Auch die sogenannte Hochliteratur, die erhabene Bildungsliteratur, wie die Wissenschaft reflektieren das Dasein immer im banalen Körper-Leben (Kiens Selbstverbrennung als Mann-Leben, aber auch Weiningers Selbstmord). Die Daten der hohen Literatur wie der banalen und trivialen Literatur gleichen sich insofern, als sie dieselben sind. Beide sind jeweils so banal wie sie jeweils erhaben sind (was aus Gründen der Ordnung zu bedauern ist).

Peter Weiss hat es in den fünfziger Jahren nicht gewagt, eine der entscheidenden Bedeutungen unseres Jahrhunderts, den Holocaust, literarisch in einem KZ-Lager darzustellen; er hat sein Werk Die Ermittlung, das nur bis an die Rampe von Auschwitz reicht, als Spiegel des AuschwitzGerichtsprozesses und als "Oratorium" gestaltet; der Bedeutungskörper KZ schien ihm literarisch nicht formatierbar, ebenso wie schon früher Theodor W. Adorno gemeint hatte, nach Auschwitz sei kein Gedicht mehr möglich. Aber: Art Spiegelman hat 1986 das Leben im KZ als MäuseKatzen-Schweine-Comic, also im Medienvergleich banal und trivial, aber für eine andere Zeitgenossenschaft, für die nicht mehr unmittelbar in das Mordgeschehen verwickelte Generation, wirkungsvoll und - durch Kritik und Preisrichter - künstlerisch anerkannt, also hochliterarisch dargestellt. In der Risiera von Triest, dem Triestiner $\mathrm{KZ}$, wurden die Originalzeichnungen auch vor den Verbänden der Überlebenden ausgestellt und anerkannt. Spiegelman "individualisiert" das Holocaust-Geschehen über ein angespanntes bis zerbrochenes Vater-Sohn-Verhältnis, also ödipal in der Geschlechtsnachfolge; er formatiert den Holocaust familial um; zusätzlich $\mathrm{zu}$ seiner kunstverhindernden "totalen" Bedeutung als Fabrik-Mord ist er 
bei Spiegelman auch die spezifische Bedeutung eines Überlebenden, dessen Überlebensform dem Sohn banal erscheint. Erhabenheit und Banalität sind relative Formen im lebensbestimmten InformationsprozeB. Redet man auf der einen Ebene, redet man immer auch auf der anderen. Was man von Sigmund Freud nicht hören wollte, als er die bewußtseinsbestimmenden Bedürfnisse des Menschen in ihren mehr oder weniger hochkulturellen Verarbeitungsformen zeigte und die Handlungsformen des Menschen auf beiden Ebenen gleichzeitig erzählte.

\section{Weiß die Kunst mehr? Sein oder Nichtsein}

Ich hoffe, daß Sie, aus der relativen Identität von Erhabenem und Bana_lem, meine folgenden Kalauer auf der erhabenen Ebene lesen. So wie ich will, daß mich die Buchhändlerin als Hochliteratur-Käufer hört, wenn ich in der Buchhandlung Elisabeth Reicharts Roman Die Fotze (1995) verlange. Nach vielen erhellenden Seiten lese ich in diesem Text, daß die Heldin alles andere lieber ist als sie Tochter und Geliebte, als sie "Fotze". ist. Es gibt ihr Geschlecht für sie jetzt nicht. Denn es gelingt unter den gegebenen Umständen nicht, es wird für andre weder zu der Information noch zu der Bedeutung, die die Heldin sein will. Es gibt nur die "Fotze", auf die zu sein sie - in elegisch-erhabener, elitärer MonologForm: sozusagen pyramidal - zu verzichten sucht. Es wird kein Mensch aus der "Fotze", der auch des andern Fleisch wäre, als Gleichnis der Gattung, die biblisch ein Fleisch ist. Demnach kann man den Buch-Titel Die Fotze in der Buchhandlung laut verlangen. Man verlangt Reichharts literarische Formatierung der Herkünftigkeit von Bedeutungen ("Frausein"). Es handelt sich, wie bei der Pyramide, um einen Definitionsversuch des Menschengeschlechts. Ich zitiere dazu den Kalauer, der mein Pyramiden-Beispiel kathedral angeregt hat. Dieser Satz des Dichters Walter Grond lautet:

Die Kunst weiß mehr. Sie kennt die Spur ins 21. Jahrhundert. Am Ende des Jahrtausends drohen die Dummen und Geistfeindlichen das Lesen und Denken zumVer- schwinden zu bringen. Die Pyra mide aber wird höher, die Konzepte sprengen den Rahmen, die Li teratur ist die Kathedrale der Intelligenz. 
Ein Satz, so idiotisch, meint man, daß er in der Werbung nicht mehr vorkäme, mit Ausnahme z. B. der Humanic-Werbung, die zeigt, daß man Waren-Bedürfnisse, ohne die Waren ("banal") zu nennen, durch Befremdung der Bedürfnisse "geistig" bewerben kann. Der Unterschied liegt in der Bewerbung, nicht im Beworbenen, wie Gustav Droysen auch für das Geschäft der Geschichtsschreibung schon 1868 in seiner Historik deutlich machte. Wir machen die Geschichte und wir machen Geschichten (entgegen der befehlenden Redewendung: "Mach keine Geschichten!"). Walter Grond will, stilistisch pyramidal redend, Geschichten machen gegen das in der Gegenwart nicht in sich unterschieden, sondern total scheinende Banale als Erhabenes.

Mit Gronds pyramidalem bzw. kathedralem Satz ist also in einer möglichen Form der Lebenskampf der "hohen" Kunst gemeint: in gegenwärtiger repräsentativer Banalität wird das Erhabene - "die Kunst" von Grond trivial behauptet (in antiquierter Metaphorik beworben). Grond, jetzt Präsident des Forums Stadtpark Graz, ist - um auf der Ebene seiner Werbung zu bleiben - der Arnold Schwarzenegger der Literatur, ein Terminator. In seinem Roman Das Feld erzählt Grond einen Menschen von dessen gegenwärtigem Leben über seine Jugend und Kindheit zurück vor die Zeugung, "löscht" ihn also - auf der Ebene des Banalen und in der Metaphorik der Unterschichtensprache ausgedrückt: er pudert ihn zurück. Er schreibt seinen Helden in der gegenwärtigen Bildungsliteratur aus ihr und aus seinem in ihr gestifteten Leben heraus. Die Arbeit des Schriftstellers vermittelt nicht ein Heldenleben an den Leser, sondern nimmt es ihm absichtlich und merkbar weg; er schreibt es ins Nicht-Leben, ins Nichtige des Nichtbestehens, ins "Ungeborene" bzw. Noch-"Tote": wohin sich Hamlet aus der Intrigen-Welt weg hinwünscht. Er schabt ihn von der Gattungspyramide. Heute heißt das: er löscht ihn cool, absolut, ultimativ auf der Super-Pyramide, auf der Mega-Pyramide, auf der UltraPyramide, mit welchen Ausdrücken sich gegenwärtiges Leben ins Erhabene gegenwärtigen Bedeutens kanonisiert. Demgemäß heißt, in Anlehnung an die Vodka-Marke Absolut, ein absolut nichtssagender Roman im Kriminal- (bzw. kriminellen) Schema gegenwärtigen Bedeutens von Walter Grond Absolut Grond. In der absurden Behauptung seines zitierten pyramidalen Satzes über die Kunst im 21. Jahrhundert, der in der Werbung nicht mehr vorkäme, ritzt Walter Grond aber zugleich andere 
Bedürfnisse seiner Literaturfiguren auf die gegenwärtige Wertungspyramide. Er baut Pyramiden, wenn auch andere. Ich nenne eine: Absolut Homer heißt sein Roman, in dem aber zwanzig Autorinnen und Autoren als Reisebeschreibung (nachgestellt der Odyssee als moderne Umreisung der Welt) den, der sie zu dieser Beschreibung aufgefordert hat, Grond, als Autor schreiben. Grond least sich Schriftstellerpärchen, die jeweils Reisen machen oder Reisen denken, zur Selbstherstellung als Autor und wird so "Absolut Homer". Homer ist eine hohe Überlieferung wie die Mykerinos-Pyramide. Ursprünglich sollte, nach einem 1992 publizierten Konzept, dieser 1995 erschienene Roman nicht Absolut Homer heißen, sondern: Grond. Ein Roman Homer-ident-Grond (hen diaphéron heauto): Erhabener geht es auch bei Mykerinos/Rhodopis nicht $\mathrm{zu}$. Und auch hier geht es um monumentale Selbstzeugung. So vielfach $20 \mathrm{fach}$ - vermittelt konstituiert bzw. zeugt sich heute ein Autor, als ein sehr relatives Spiel der Bedeutungen ausgewählter anderer (Frauen und Männer). Die Zeugung eines Autors, das Autoren-Geschlecht, der Musenkuß, ist augenscheinlich ein schwieriges Geschäft geworden. Von einem "Ich" - seit Ernst Mach in den bürgerlichen Formen "unrettbar" - als literarischem Auctor ist nur mehr vermittelt, nicht mehr eigentlich die Rede, ist überwiegend durch andere die Rede. Nicht nur der Held, auch der Autor ist nicht mehr selbstverständlich, sondern ein bestimmtes Format anderer und seiner selbst.

Gewiß hat eine solche Selbstherstellungs- und Selbstdarstellungsschwierigkeit historisch-konkret viele Gründe. Einer aus ihrer Vielzahl im vorliegenden Zusammenhang des Geschlechts:

In Arnolt Bronnens Familiendrama Recht auf Jugend aus dem Jahre 1913 sagt der Vater zu seinem Sohn: "Was is so n Kind? 'ne klebrige Sauce, die ich anderfalls in mein Präservativ gewischt hätte". Kein Wunder, daß man sich, bei so fahrlässiger Geschlechtspraxis der Väter und solchem Lebensentzug für die Söhne, eine andere Zeugungsform sucht, im Zeitalter der Pille bzw. der legalen Abtreibung z. B. eine selbstgewählte Elternschaft wie der "Autor" Grond.

Die Verhältnisse scheinen im Urbild der Bedürfnisse, im Geschlechtlichen, seit Bronnens Drama nicht besser geworden zu sein: "[...] du hättest mich auch in den Abort scheißen können", verweigert sich und ihr mißachtetes Frauengeschlecht eine Tochter der Mutter in dem Roman Die Zuichtigung von Anna Mitgutsch. Vergleichbare Selbstdefinitionen fin- 
den sich auch bei männlichen Gestalten. Die Ich-Erzählerfigur durch vier Romane Josef Winklers, der Enznsepp, tritt zumindest die Geschlechtsnachfolge des Vaters nicht an: "So lang Du lebst, mein Vater, verzichte ich auf mein Geschlecht". Und er beschreibt seine schwule Praxis. Die Beispiele lassen sich vermehren (ohne dadurch im Rahmen so vieler literarischer Sätze repräsentativ zu werden). Augenscheinlich eine prekäre Situation, die auf den Körper bezogen und bezogen auf seine Herstellung in pyramidaler (Bildungs-)Literatur ausspricht, daß besser nichts sei, als das, was ist und was unter den gegenwärtigen Bedingungen der Herkunft sein kann. So spricht Hamlet von Sein oder Nichtsein.

Der - wegen der hohen Kosten - vielkritisierte, keine Herkunft schaffende, für viele banal-unwirkliche Telefon-Sex, der aber, nach seinen Frequenzen, augenscheinlich die Verarbeitung der Geschlechts- informationen für viele andere ausreichend leistet, stellte vielleicht auf der erhabenen Ebene eine pyramidale Lösung dar. Aber der gegenwärtige Körper ist nicht immer mit solchen erhabenen Lösungen solidarisch zu machen. In Marlene Streeruwitz' Drama Wakiki Beach, das wie Schwabenitzkys Film Verlassen sie Ihren Mann, einen lokalen Wahl-kampf mit Vermarktung auch der "Frau" des Kandidaten zum Stoff hat, sagt die insgesamt auf ihre Abbildung als Frau verwiesene Frau: "Wie soll man einen Fick am Telefon von einem andern unterscheiden. Auf Band. Ich frage dich". Sie will sich zumindest im Geschlechtlichen anders, "wirklich", als "ein Fleisch" mit dem andern Geschlecht erschei-nen.

\section{Sanktionen}

Der Körper, der wir sind, ist eine in unterschiedlichen und komplizierten Strukturen organisierte Masse, wie die Schul- und die Alternativmedizin zeigen. Sie ist organisiert und sie wird rückwirkend organisiert durch das, was sie selber produziert: Bedürfnisse, die der Körper entläßt; die er ungestalt entläßt, wenn sie im Rahmen unserer Konvention nicht wahrnehmbar sind oder nicht wahr genommen werden (sollen), oder die er in die Gestalt von Empfindungen, Vorstellungen, Bildern, kollektiven Übereinkünften entläßt (also insgesamt: in schon etablierte Körperabbilder entläßt). Pyramiden sind ein nur noch verschwindend kleiner Teil davon (der Präsident Frankreichs, François Mitterand, ließ [sich] eine bauen im 
Hof des Louvre als Eingang zu einem ausgedehnten unterirdischen Röhrensystem, in dem die Besucher - die Nahrung des Museums in seinen Gedärmen - vor die monumentalen Kunstwerke geführt werden. Mitterands Pyramide und Röhren usurpieren die Erhabenheiten der Weltkunst für den Präsidenten; er verewigte sich. Röhren wie von einem nach außen gestülpten Leib kennzeichnen den Bau, in dem sich ein Vorgänger Mitterands, Georges Pompidou, als Centre Pompidou verewigte. Beide Präsidenten wußten zum Zeitpunkt des Baus von ihrer Todeskrankheit. Repräsentativer für unsere Zeit als diese pyramidalen Unternehmungen ist aber ohne Zweifel die Pyramide des Spielcasinos Luxor in Las Vegas).

Sensible kriegen aus diesen Wechsel-Verhältnissen zwischen Bedürfnissen und den Bildern für sie z. B. die Magersucht oder die Fettsucht, ebenso typische, aber vorbewußte, aesthetisch nicht-etablierte Reaktionen. Wie "ein offenes Rasiermesser" rennt Woyzeck bei Büchner durch die Welt unter dem Druck des Geschlechts und des ihn empiristisch behandelnden Doktors, der ihn in Ursachen und Wirkungen zerlegen will. Woyzecks Körper nimmt als Rasiermesser die Verhältnisse wahr, der Körper Maries hat es zu leiden. Der Körper reagiert auf seine Gedanken bzw. auf die Gedanken der Körper-Gattung "Mensch", auf ihre Bauten und ihren Fleiß, auf ihre Pyramiden und Industrien. Die Allergien nehmen zu und angeblich die Spermiendichte ab: körperlich reagieren wir auf die z. B. in der Industrie schon verwirklichten Bedürfnis-Ausscheidungen unseres Körpers. Ein somatopsychischer, psychosomatischer Sanktionsmechanismus eines Einen in sich unterschiedenen. Sinnfrage und Existenzfrage können also nicht gegeneinander ausgespielt werden, sondern sind vermittelt ident, so wie die Literatur in ihren Formen, in der Nutzung ihrer Gattungen, sich herstellt, im Prinzip - immer noch Schiller-klassisch als "Aufhebung" des sogenannten Stoffs im Format.

Auf der Voraussetzung des Körpers denken wir uns und ihn, d. h. er denkt sich, wenn wir uns denken. Er ist immer schon da, und zwar herkünftig-unzureichend.

Nach der Genesis schuf Gott die Menschen als "Menlin" und "Frewlin", aber als "ein Fleisch", und nannte das nach Geschlechtern unterschiedne eine Fleisch "Mensch". Er schuf sie nach der Luther-Übersetzung als sein "Gleichnis". Aber er stellt es unter einen Sanktionsmechanismus, um auch er selber, Gott, Auctor, bleiben zu können. (Es könnte ja, nach 
Musil, ebensogut anders sein). Das "Gleichnis" sollte sein Geschöpf bleiben und (ihn) nicht erkennen, wohl in jenem auch schöpferischgeschlechtlichen Sinn, den das Wort "erkennen" in der Bibelübersetzung hat, nämlich "beiwohnen", "beischlafen". Gott wollte sich sein Gleichnis vom Leib halten bzw. als nicht-leiblich in unserem Sinn gelten. Die Fruchtnutzung am Baum der Erkenntnis wurde untersagt. Der christliche Sanktionsfall gegen die Erkenntnis tritt durch das Unterschiedensein des "einen Fleisches" ein, durch Eva, die - als Teil Adams sein Unterschiednes - von der Penis-Schlange verführt, Adam Erkenntnis verschafft und erkannt wird. Das Fleisch ist in der Bibel die Erkenntnisform.

\section{Einzigkeit, pyramidale Ambivalenzen, ihre Motorisie- rung und der Arbeitsmarkt}

Die unfriedliche "Fortsetzung" des Geschlechts - altgriechisch z. B. in Gestalt des Oidipos bzw. neueuropäisch in Gestalt des "ÖdipusKomplexes" - kennt die Bibel z. B. in der Konkurrenz der Söhne Kain und Abel. Kain erschlägt den Bruder Abel, um allein - nicht als Unterschiedner in der einen Gattung - die Liebe des Vaters zu gewinnen. Der Einzige in der Gattung kann aber nicht sein Eigentum sein.

Kains Wunsch nach Einzigkeit liegt nicht ab. Ein Beispiel aus der Sachund zugleich Innerlichkeitsliteratur: der ultimative Mega-Bergsteiger auf den Mega-Bergen Asiens, Schriftsteller und Philosoph Reinhold Messner, der sich selber altgriechisch als Sisyphos definiert, gesteht sich Kains Sehnsucht, die unterschieden zweigeschlechtige Gattung mit seinem Geschlecht zu monopolisieren, an sich selbem ein (oder wirft sie sich vor) in der Sehnsucht nach seinem Wunsch-Kind (unabhängig von den Kindern, die er hat):

Leider ist ein Kind aus mir selbst heraus nicht denkbar, und ich möchte kein Kind von einer Frau, kein Kind, das von zwei Menschen abhängt. Das eigene Kind aus mir selbst als Idee und doch gleichzeitig als rational nicht greifbare Vorstellung,

beschreibt er seine Bedürfnisse, ein zweigeschlechtiges Fleisch zu sein, und motiviert zugleich seine imponierenden - pyramidalen - Gipfelstürme 
als Sisyphos. Denn die Gipfelsiege sind Messner als sein Kind aus sich und als seine Einritzung in die (Berg-)Pyramiden. "Die letzte Chance ist Bewußtsein", erkennt die Doppelfigur Alexander (der Große und ein Abenteurer der Gegenwart gleichen Namens), als er - in Arnolt Bronnens Stück Ostpolzug 1926 - den "Ostpol", den Mount Everest, zu ersteigen versucht (1926 "noch eine Sache von Wert [...] mit der Haut einer Jungfrau"), um "endgültig den Geruch der Menschen" zu verlassen: "Ich bin dagegen, $\mathrm{Daß}$ selbst, wo ich abstürze, andere schon längst abgestürzt haben." Auch er will einzig sein. Dem trägt Bronnens Drama als Einpersonen-Stück Rechnung. Doch ist die eine Person als Doppelfigur Alexander (der alte)/Alexander (der neue) verdoppelt-uneins und im pyramidal-himalayesken Diskurs historistisch. Zudem fühlt Alexander sich zugleich als Frau (Roxane) und fühlt, "daß ich lieber nicht so allein wäre", als er "Auf dem Gipfel des Mount Everest, 8840 Meter, Mit meiner Nase noch einen Meter darüber", steht, mit diesem Gipfelsieg sich zeugend wie Messner sein Kind:

Das war der Ostpol, das war die unbesiegbare Erde, Hier, wo ich stehe, Religionen unter meinen Füßen, [...]

Aber doch etwas höher, als es mir bestimmt war, Doch etwas weiter, doch etwas stärker, doch etwas ewiger Als das Schicksal es in meine Hand schrieb [...] [...]

Manche Wünsche werden Jahrtausende alt, Aber unaufhaltsam wachsen die Organe ins Unsichtbare hinein,

Und der Gewinn der Unsterblichkeit ist nahe.

Es geht - und das wurde in der bronnenschen Form nicht zu unrecht als faschistisch bezeichnet - um die Einzigkeit, die Unsterblichkeit, um die Pyramide, die gewiß ein ambivalentes Menschheitszeugnis ist, so trivial wie erhaben, Lebenswunsch wie Todeszeugnis. Bronnens abendfüllendes Ein-Mann-Drama ist Selbstzeugung als Text, als großer Dichter im Spiegel des großen Reichsschöpfers Alexander; der Autor will sich als Auctoritas erkennen. Autor heißt: sein Geschlecht (nicht zweigeschlechtig) "erkennen", sich als sein Kind zeugen. 
Was derzeit bleibt, ist, weit unter Messners Gipfeln, ein irdisches Jammertal, in dem zeitgenössisch volle Autobahnen zu Raststätten führen. In deren Toiletten werden, nach dem Stück Raststätte von Elfriede Jelinek, die vorher in Zeitungen mega-super-ultra-hyper - also vermittelt/bildhaft/gattungsspezifisch - annoncierten Menschen-Ge-schlechtsBedürfnisse so gut wie mega-ultra-super-hypermöglich voll-zogen, gefilmt und nachkontrolliert. Sie gelingen in dem Stück Jelineks aber trotz dieser gattungsbewußten, informationsreichen Vielfalt an vermittelnden Bildern - so wenig wie die Gattungsansprüche in Reicharts Fotze eingelöst werden: die "wahren" Bedürfnisse, "daß wir endlich hochspringen und von uns wegkommen", daß wir hochspringen zum "einen" Fleisch der Gattung, diese Gipfel-Bedürfnisse werden im Stück nicht zufriedengestellt, auch wenn der Liebe "Motor auf Touren kommt" und "bis zur Mortalität hochgejagt" wird. Der "Lendenwagen" des Mannes und der "Kleinwagen" der Frau können nicht - wie in der griechischen Mythologie bei Salmakis und Hermaphroditos - synchronisiert werden: "Diese Frau hat schon seit Jahren ihren Rückwärtsgang im Buch des Lebens eingelegt, was erwarten Sie noch", heißt es in Jelineks Roman Lust, einem wild aus auch männer-pornographischen Vorgaben montierten Werk. Die banale Rückanwendung der Terminologie der motorisierten Produkte-aus-Körperbedürfnissen auf eben diese Bedürfnisse scheint eine Vanitas vanitatum vanitas solcher Formatierungen zu signalisieren. Sie müssen schlecht gelingen, solange es zwei Geschlechter gibt und nicht alles eins ist.

Das Stück war, zu unrecht scheint mir, für die Süddeutsche ein Skandal. Vielleicht aber ist es ein Skandal für die Arbeitsmarktverwaltung: Jelinek banalisiert die hochrangigen industriegesellschaftlichen Ausprägungen des Geschlechts, so daß wir uns nicht in ihnen erkennen wollen. Jelinek gefährdet damit Arbeitsplätze. Denn die industrielle Materialisierung der Bedürfnisse bietet, bei immer weniger werdender bezahlter Arbeit, zahlreichen Menschen in der technischen, medizinischen und psychologischen Forschung, in der Beratung, Mode, Werbung, in der Design-, Auto-, Bild, Film-, Unterhaltungs-, Medien-, Sport- und Pornoindustrie einen gewissen Kündigungsschutz, bringt vielleicht in Zukunft vermehrt Arbeitsplätze, wenn keine andern Regressionen unserer Kultur - denn nach Sloterdijk sind Alternativen Regressionen - als die auch am Auto sichtbaren eintreten. Das unbefriedigte "eine" als nicht-eine Fleisch braucht 
immer größere materielle Abbilder und bringt sie hervor. Jelineks Stück ist in diesem wirtschaftlichen Sinn als ordinär empfindbar.

\section{Erhabenheit als (demoskopische) Erhebung des Banalen. Pyramidale Ko-Texte und parisitäre Literatur-Texte}

Die Raumaufwendigkeit von Pyramiden, Kathedralen, Fahrzeugen, Autobahnen, Emissionen und anderen Vergegenständlichungen der getrenntgeschlechtlichen Bedürfnisse ist in der industriellen Massen-gesellschaft bedeutend. Die Abschaffung selbst ihrer Verpackungen - der "Sorgen" nach dem Begriff der Entsorgung - macht Probleme.

Einer der größten gegenwärtigen Raumaufwände ist der Ton-Raum im Xlargen open-air-Live-Alltag als durchdauerndes Event. Das gilt auch für die "hohe" politische Selbstdarstellung: die 1000-Jahrfeier der ostarrîchiUrkunde, die die österreichische Regierung im Marktflecken Neuhofen in Niederösterreich als Staatsfeier abhielt, brachte ein Open-air-Konzert der sogenannten Austropop-Stars; zu dieser Zeit wurden - ohne viel Lärmim Parlament in Wien Spar-Gesetze im Verfassungsrang (und gegen die Bedenken der Verfassungsrichter) beschlossen, um sie unanfechtbar zu machen: zwei sich ergänzende Formen der Massen-Moderation. Die Tiroler holen Tina Turner, damit sie in den Tiroler Bergen in 2.500 Meter Höhe ein Open-air dröhnt, während die Tiroler bei der europäischen Verkehrskommission gegen den Transit-Lärm protestieren. In Linz wird ein repräsentatives Kultur-Festival mit einer "Klangwolke" abgehalten, bei der Bruckner aus allen Radioapparaten und allen andern möglichen Verstärkeranlagen gedröhnt wird. So lehrt Kulturverwaltung und Elektronikindustrie das Volk Kunstmachen. Usw.

Schon vor der elektronischen Datenverarbeitung auf dem Ton-Bereich hat vergleichbarer Ton-Raum in diesem Jahrhundert Triumphe gefeiert: als die Verstärkeranlagen noch in den Kinderschuhen steckten, haben die Hitlerund Goebbels-Reden, die auch geschlechtliche Vorgänge waren, die Bedürfnis-Massen nach einem andern Dasein ins Unmeßbare (z. B. ins "Pyramidale" der "Deutschheit") gesteigert (und dieses Unmeßbare dann tödlich an dem, was ihm nicht zu entsprechen schien, gemessen). Robert Musil sah damals Hitler als "Christus mit Radio", Ernst von Salomon hörte Hitlers rundfunk-adäquate Rede-Modulierung als "technischen" Griff 
in die Tiefen des Menschen. In Ernst Jandls Laut-starkem Gedicht Wien Heldenplatz, dem Ort von Hitlers Siegesfeièr in Österreich 1938, durchsetzt dieser Lärm die Wortnormen und wird darin diagnostiziert:

der glanze heldenplatz zirka versaggerte in maschenhaftem männchenmeere drunter auch frauen die ans maskelknie zu heften heftig sich versuchten, hoffensdick. und brüllzten wesentlich.

verwogener stirnscheitelunterschwang nach nöten nördlich, kechelte mit zu/nummernder aufs bluten feilzer stimme hinsensend sämmertliche eigenwäscher.

pirsch!

döppelte der gottelbock von Sa/Atz zu Sa/Atz mit hünig sprenkem stimmstummel. balzerig würmelte es im männechensee und den weibern ward so pfingstig ums heil zumahn: wenn ein knie/ender sie hirschelte.

Der Maßstab für die durch Jandls Gedicht brüllzenden Geschlechts-Massen von 1938 ist das, was durch sie vergewaltigt wird: In Thomas Bernhards Drama Heldenplatz (1988) dringen die auf dem Wiener Heldenplatz der Hitler-Rede antwortenden Ton-Massen der österreichischen Nazis des März 1938 der Figur 'Frau Professor' - die aus der Emigration nach Wien zurückgekehrt ist - immer noch ins Ohr, obwohl sie jetzt real nicht mehr laut tönen (und natürlich nicht im Stück). Ihr Körper nimmt sie immer noch wahr; sie wird ständig durch ihr Ohr vergewaltigt, schließlich getötet. Ihr Kopf fällt in den Suppenteller. $I h r$ Sprechtext hat gegenüber diesem Lärm keine Chance. Bernhards Heldenplatz-Figuren sprechen ihre beeindruckenden monologischen Texte im Banalen ihrer Chancenlosigkeit im österreichischen Lärm. Ihre sprachliche Intensität ist zugleich ein Verstummen vor ihm.

Es ist jetzt immer März 1938, was den Ton-Raum betrifft. Im Gastgarten Gretl in Krumpendorf am Wörther See war im Vorjahr der täglich mehr- 
mals wiederholte Höhepunkt der plärrenden Saison ein Potpourri aus folgenden Lied-Dichtungen: "Wir wollen bumsen, bumsen [...]; Ein schöner weißer Arsch, mit einer bunten Feder drauf [...]; Wir trinken das schäumende Bier und scheißen dem Wirt auf die Theke [...]". Es geht bei diesem Chor-Lärm nicht primär um Unterhaltung im Fremden-Verkehr; es spielt sich etwas anderes $a b$, etwas allen Eigenes: Eigen-Verkehr, kollektive Selbstzeugung im begeisterten Gebrauch vulgärer Worte, ein Sich-Pudern. Es spielt si ch etwas ab - mit Jandl: - bei "sämmertlichen eigenwäschern". Krumpendorf ging inzwischen auch durch die deutsche Presse, weil dort der österreichische Politiker Haider bei einem Treffen der SS-Kameradschaft IV die Anständigkeit der Teilnehmer rühmte. Der Krumpendorfer ÖVP-Bürgermeister schrieb in der Gemeindezeitung: die SS-Kameradschaft IV sei "immer mehr zu einer Friedensgemeinschaft geworden". Soviel zum Lärm-Kontext Bernhards und Jandls heute.

Jandls Gedicht Heldenplatz zeigt die geschlechtliche Entsprechung zum schönen Lärm der (damals nationalsozialistišchen) Begeistèrung bžw. Geisterfülltheit, in der das uneine Fleisch im religiös-pfingstigen Eigenwäscher-Brüllzen als Gehirscheltwerden durch den "stimmstummel" eins $\mathrm{zu}$ sein schien und glücklich war, und zeigt so auch den Untergrund gegenwärtiger (zeit)geisterfüllter "schöner" Mega-Stimmung.

Der laute Banal-Text der sich erfüllend verstehenden Wirklichkeit - sei's am Heldenplatz, sei's in Krumpendorf, sei's bei der Regierungsfeier in Neuhofen - bedrängt, durchdringt, überlagert und macht den poetischen Text wie der "stimmstummel"-schöne Lärm Jandls Gedicht macht. Douglas Coupland - um das Land zu wechseln - zeigt das graphisch: die Randzeichnungen (z. B. die "veal-fatening-box") und das Mainstream-Randglossar in seinem Roman Generation X. Tales for an Accelerated Culture (1991) ersparen dem Autor viel traditionellen Romantext. Es braucht den alten Romantext in der (Best-)Seller-Welt der Standard-Texte nur mehr als subversiven Kontrast. Er verhält sich zu den Standard-Vorgaben wie ein Parasit.

Michael Chabons Wonder Boys (1995), ein anderes Beispiel aus der Bestseller-Welt, beschreibt die Schwierigkeit bzw. die Eigenart des modernen Verbrauchs des traditionellen poetischen Textes ausführlich: Ein weit gediehenes Romanmanuskript Wonder boys soll - innerhalb 
der Romanhandlung des Romans Wonder Boys - fertig geschrieben werden, d. h. traditionell: "das Werk", das den Autor für ihn selber und für den Verleger und für den Leser schafft. Das Manuskript dieses im Roman Wonder Boys besprochenen Romans Wonder Boys, um dessen Fertigstellung es geht, geht aber in der Romanhandlung zugrunde: $\mathrm{Da}$ es keinen Roman Wonder Boys gibt, erscheint als Roman Wonder boys. "Das Werk" gelingt nicht mehr "für sich" in der "accelerated culture" des Verlags-Bedarfs; es gelingt nicht mehr vor dem nächsten Bestseller, der es, noch unfertiges Werk eines Autor-Schülers, aus dem Verlagsvertrag drängt. Der "Autor" kommt nicht mehr dazu, sich als Autor herzustellen, sich zu zeugen. Die Fiktion "rundet" sich nicht mehr zum "Werk" in der Marken-, Verlags- und Intellektuellen-Wirklichkeit, deren eigener KunstCharakter die Fiktion dynamisch aufwiegt bzw. überholt. Der Autor, der sich endlich, nach Jahren, mit der Fertigstellung der Wonder Boys als Autor nachweisen will, ist keiner, schafft das Werk nicht und damit nicht sich selben als Autor: Daher wohl machte Grond so gewaltige Umstände, um Grond zum Autor werden zu lassen: von Absolut Grond zu Grond. Ein Roman und zu Gronds und anderer Absolut Homer. Und daher will wohl der Medien-Bergsteiger Messner sein Kind.

Dazu zwei Hinweise, einen auf die Tradition, einen auf die Gegenwart. Die bürgerliche Hochliteratur - nicht nur ihre Avantgarde - hat schon lange auf diese Spannungen zwischen Roman-Text und andern hochentwickelten Ko-Texten (K. Schuhmann) der Wirklichkeit hingewiesen: Musil, der im Mann ohne Eigenschaften die so gewalttätige wie schöne Fiktionalität der bestehenden Wirklichkeit im Adels- und Bürgerformat zum Ausgangspunkt nahm, wollte seinen nicht fertigen Roman-Text im Zweiten Weltkrieg, also im Schweizer Exil, folgend abbrechen: die problematische Substanz sollte in Essay-Bände ausgelagert werden, der Held des Romans aber, in pirandelloscher Ironie, ein Schlußwort über den Autor schreiben. Wollte sich auch Musil, Autor der Weltliteratur, zurückpudern in eine fiktionale Nicht-Auctoritas, um den (ohnehin schon "negativen") Helden zu retten, wenn schon der Autor im Ko-Text des Kriegslärms und der Exilstille verloren geht? Elias Canetti plante um 1930 eine achtbändige Romanreihe als "Comedie humaine an Irren". Sie wird - nach dem ersten Roman Die Blendung - abgebrochen bzw. mutiert unter dem Eindruck gesellschaftlicher Gewalt zum Essay-Werk Masse 
und Macht. Kein Roman folgt. In der gewaltigen Textanstrengung umfänglicher autobiographischer Schriften rettet sich Canetti, der Autor eines poetischen Frühwerks, als poetischer Dichter. Ist diese Rettungsaktion mit dem Nobel-Preis belohnt worden? Der Förderer Canettis, Hermann Broch, beschäftigte sich mit der "epischen Integration", also mit dem Verhältnis von "realen" und "fiktionalen" Texten, nicht anders "neusachlich" Edlef Koeppen, Ernst Jünger, Rudolf Brunngraber, Arnolt Bronnen usw. - ganz abgesehen von Formen des DADAismus -, bis Handke die Aufstellung einer Fußballmannschaft als poetischen Text druckte und bis schließlich - ich fasse sehr gewaltsam zusammen - bei Oswald Wiener, in der verbesserung von mitteleuropa, roman (1969), Unterscheidungen wie fiktional/nichtfiktional hinfällig werden. Nicht nur die Werbe- und die politischen Sprachen, auch die Wissenschaftssprache, die ebenso die poetischen Texte eher bedrängt als beschreibt, werden von diesem gewaltigen Text Wieners noch einmal stumm gemacht. Die Problemlage besteht fort in einer "Ultra light-last minute-ex+popliteratur", nicht zuletzt in der Theorie der Frauen-Sprache, der Kathy Acker in den gegenwärtigen Verhältnissen die Mimesis-Fähigkeiten abspricht.

$\mathrm{Da}$ die Funktion des Autors (der im Werk sich zeugt) relativ ist zu anderen Texten, zeigt sich traditionell-gattungsmäßig z. B. in der Parodie also im Rahmen der werkkonstituierenden Dichtungsgeschlechter der Gattungen. Als Parodist ist Christian Wallner in den 70er Jahren in die triviale Kioskliteratur weggetaucht und hat für den Bastei-Verlag Trivial-Parodien geschrieben wie Das Glück ist ein Suchen. Roman einer ChefSekretärin, die über den normalen Heftchen-Vertrieb liefen und also vom typischen Leser-Publikum des Bastei-Verlages "normal" konsumiert wurden, als Autor-Text demnach unerkennbar blieben. Der Text Wallners ist potenziert trivial, eine Lesefrucht unzähliger Trivialtexte, und ist darin so dicht, daß ich ihn nur als Hochliteratur lesen konnte. Für mich wie für den Kioskliteraturleser ist er Hochliteratur aus dem Geschlecht der Trivialliteratur.

Man kann, im Rahmen dieser Literatur- bzw. Sachlage gleich auf die anspruchsvollen traditionellen Bedeutungen "Werk" und "Autor" verzichten oder sie zumindest minimieren, wie es mir z. B. die Texte von Kapielsky (ohne Vornamen!) zu tun scheinen: Er gibt nur Lomos, Bildund Text-Lomos, die in dem Buch Aqua botulus in ihrer Bedeutungslosigkeit dicht aufeinander bezogen werden. Das Werk Der 
Einzige und sein Offenbarungseid gibt als Autor bezeichnenderweise "Kapielsky und Freunde" an und heißt im Untertitel Verlust der Mittel.

Die Texte der dichterischen Tradition wie der gegenwärtigen Wirklichkeitsbewerbung werden von solcher Poesie nicht mehr konkurrenziert oder überboten, sondern unterboten. Der neue Autor-Text soll etwas heißen, indem er nicht viel heißt. Der Text, könnte man sagen, ist traditionell gesehen parasitär an Vor-Texten bzw. an der Tradition - parasitär im Verständnis der Biologie, die das Parasitäre positiv definiert als Verzicht auf Vollständigkeit durch Entsprechungen und Bezüge. Dieser Verzicht wird biologisch auch als Regression bezeichnet. Auch in diesem Sinn sind, wie Sloterdijk meint, alle Alternativen regressiv bzw. eine andere Form von Regression als die etablierte. In der Literaturgeschichte, aber auch in der gegenwärtigen Literatur-Betriebssituation sind eine Fülle von solchen "Regressionen", d. h. parasitären Benützungen, Korrumpierungen verfügbarer und geltender Schemata, zu beobachten.

Der Detektiv- bzw. Kriminalroman des ausgehenden neunzehnten Jahrhunderts (Sherlock-Holmes-Typus), wahrhaft Massenliteratur, wird neben seinem mächtigen Weiterleben auch in FS-Serien - in seiner positivistischen Struktur "reggressiv" korrumpiert z. B. durch jene PulpFiction, wie sie als Typus Quentin Tarantino in Pulp Fiction (Buch und Film 1994) faßte: "Bei Titel und Handlung ließ sich Quentin Tarantino von den populären Krimi-Heften der dreißiger und vierziger Jahre inspirieren, die auf weichem, schäbigem Papier - pulp genannt - gedruckt waren. Autoren wie Dashiell Hammett, Cornell Woolrich, James M. Cain und Raymond Chandler" würden verarbeitet, womit Tarantino sich ich zitiere aus dem Rowohlt-Werbetext - "endgültig als radikaler und innovativer Filmemacher von Weltformat etabliert" hat. Das "Weltformat" besteht im witzig-zynischen, zusammenfassenden Digest der Klischees, im Unterbieten der Tradition mit einem kompakten, alles resumierenden Traditions-Sonderangebot, d. h. zugleich in der Entwertung und Korrumpierung der Tradition; das "Weltformat" besteht aus "Entsprechungen" im Gegensatz zur sogenannten "ursprünglichen" Textkonstitution durch das "Original-Genie" (nach alter Dichter- oder Filmemacherideologie). Peter Handke hat das 1967 in seiner Hohl-Form des Kriminalromans Der Hausierer als System beschrieben.

Ich nehme Beispiele aus den Kriminal-Serien des Haffmann-Verlags: 
einerseits wurde bei Haffmann das Werk von Arthur Conan Doyle, also der typenbildende Sherlock Holmes, schöngebunden verlegt (und inzwischen an den Heyne-Verlag abgetreten), anderseits werden von Haffmann die "Parasiten" an diesem publikumswirksamen Typus vertrieben, die, als solche parasitäre, Bestseller sind. Bei Kinky Friedman entbehrt die Herausfindung von Verbrechen und ihre Verfolgung durch den Detektiv Kinkster (Gangster?) aller kriminalistischen Logik oder erhält sie nur zum Schein, ist insgesamt aber tief in die antipositivistische Whiskey- und Abfallwelt Mannhattans versenkt, läßt die Ursache-Wirkung-Kriminologie der Tradition des Denkers und Nachmessers Sherlock Holmes hinter sich und schließt an den Raucher, an den Drogen-Genießer Sherlock Holmes an (der Alkoholgenuß Kapitän Haddocks und zeitweise auch Struppis in Hergés Tim und Struppi demonstriert die Gefährdetheit des positivistischen Wirklichkeitsbildes durch Drogen auch in der KinderComic-Literatur). Gronds an der Kriminallogik orientierter Vodka-Roman heißt nach der Vodka-Firma 'Absolut' Absolut Grond (oder Neil Barretts Alko-Krimi Pink Vodka-Blues.) Es findet eine Art Alkoholisierung des traditionellen Texttyps statt. Der rationalistische Kriminalroman-Typus der "Anatomie eines Mordes" (wie ihn bei Agatha Christie Hercule Poirot vertritt) wird korrumpiert. Die männliche Detektiv-Erfolgsstory (die Julian Barnes in seinen Kavanagh-Romanen überraschenderweise zunächst homosexuell variiert, aber dann langsam wieder ins Männliche zurückschreibt) ist dabei reduziert und ist weitab von einer kritischen Erfassung des Verbrechens oder des Gesetzes. Bei Kinky Friedman wird die Wirklichkeitskritik noch angedeutet in der Musiker-Vergangenheit Kinksters bzw. seiner früheren "Odyssee"(!) als Entwicklungshelfer, als noch sein "Scrotum" nach Südostasien abflog - Kinkys engagierter CountrymusicBand und seiner Detektiv-"Chefpartie" Ratso, Rambam usw. folgen Kurt Westbahns/Günter Brödels Kriminalromane, für die "Die Schmetterlinge" und die Chefpartie des "Trainers", des "Doc" usw. den Hintergrund banalen Erzählens und seiner Philosopheme bilden.

In Österreich begann diese parasitäre Kunst-Form in den 70er Jahren mit Christian Wallners Buch-Serie Schweigen ist Blei und in den 80er Jahren die TV-Serie Kottan ermittelt von Helmuth Zenker, einer der erfolgreichsten Literatur- und Filmschriftsteller und ehemals - so wie Kinky Entwicklungshelfer war - Mitbegründer der neo- bzw. sozial-realistischen 
Zeitschrift Wespennest. Zeitschrift für brauchbare Literatur. In Kottan ermittelt von Zenker geht es nicht um das Verbrechen in der Gesellschaft, die der traditionelle Kriminalroman in ihren Regeln (den Gesetzesnormen) spiegelt. Zenkers Polizei - der Präsident, die ganze Abteilung Kottan - sind mit ihren eigenen Neurosen beschäftigt. Die Polizei ermittelt mit interesselosem Wohlgefallen am Fall eigentlich gegen sich selbst und gibt im Fiktionalitätsbruch (z. B. in den integrierten Fernsehszenen mit der ORF-Sprecherin Chris Lohner) auch dieses sozialpsychologische Ermittlungsverfahren preis. Auch dabei fehlt ein Hinweis auf die Geschlechtsrollen nicht: Die Rolle der Frau Kottans bleibt in der Serie gleich besetzt, die männlichen Darsteller wechseln, bleiben aber als FotoGalerie der Ehemänner der Frau Kottan anwesend. Die Erfolgsserie (die 1996 wieder lief) löst sich in Szenen mit Play-back aller möglichen Erfolgsongs durch praktisch alle tragenden Rollen der Serie auf, bis keine (Kriminal-)"Handlung" - schon von der Filmlänge her - mehr eintreten kann. Es bleibt also der scheinprofessionelle Play-back-Lärm.

Zenker hat diese Pulp-Momente des Kottan ermittelt in Tohuwabohu fortgesetzt (und Oliver Baier in Monte Video daran angeschlossen). Tohuwabohu macht den Anschein, keinen Autor - auch nicht einen kompliziert wie bei Grond im Pärchen-Leasing sowohl hergestellten wie negierten - zu haben und ermangelt aller gewohnten Perspektive-Formen, die durch eine rasante Schnitt-Technik ersetzt werden. Die Entwitzung der Witze, der Verzicht auf Kamera-Hilfsperspektiven für den Zuseher, der Abbruch jeden Zusammenhangs, der Dilettantismus der Darsteller (darunter der Formel-1-Fahrer Gerhard Berger, Jazz-Gitti, erfolglose Schlagersänger, FS-Moderatoren, der Paradeintellektuelle Franz Schuh), die banalsten Witze u. a. sind formale Momente der Dekonstruktionsfähigkeit dieser Kunstform Tohuwabohu als erhabener. In der bildenden Kunst sind schon früher vergleichbare Formen des Banalen auf dem internationalen ästhetischen Markt erhaben in Erscheinung getreten (von Duchamps' ready mades zu den Güssen Segals, dem Foto-Realismus, den Video-Installationen Nam June Pikes usw.).

Zehn Jahre nach der Kottan-Serie tritt ihr Schema in der Realität als eigentliche Struktur des österreichischen Polizeiapparates in Erscheinung. Weder für die Briefbombenserie noch für die Ermordung von vier Romas durch eine Sprengladung konnte ein Täter ermittelt werden. Es wurde, in Jahren, ein "Täter-Profil" erstellt. Dabei blieb es. Die leitenden Beamten 
der Ermittlung wurden - wie die Darsteller Kottans - mehrfach ausgewechselt, ohne Erfolg. Die wirkliche Mordserie tritt als wirkliche Neurose der Polizei in Erscheinung - der Polizei-Apparat ist ein Text, den es als literarische Korrumpierung längst gibt. Die Polizei braucht keine gesellschaftliche Wirklichkeit, sie ist ihr eigene Fiktion (arbeitet sozusagen an ihrem Maastricht): Mit sich beschäftigt und in sich vertieft nach Rilkes "Ungeheurem" im Gedicht Der Stifter - stiftet sie sich selbst. Darüber hinaus gibt es keinen Täter und keinen Fahnder.

Gottes Gedanke bei der Schöpfung: "es könnte ebensogut anders sein" heißt auch bei Musil: es ist schon jetzt eigentlich alles anders als es erscheint und als das derzeit noch gültige Schema als "real" zeigt. Die sogenannten "hochrangigen" geistigen und gesellschaftlichen Verwaltungssysteme und die Vermittlungsformen werden in ihrem (natürlich nicht ständig, manchmal aber auch überhaupt nicht nachweisbaren) HochWert korrumpiert, sind in diesem Sinn (kaputte) Literatur (parasitär an der Literaturtradition). An der relativen Identität von Parodiertem und Parodie, an "korrumpierenden" Vermittlungsformen, sind immer schon Größenwechsel deutlich gemacht worden.

Die Korrumpierleistung der literarischen Modelle ist dabei keineswegs immer deutliches Programm wie in Kottan. In Robert Menasses Roman Schubumkehr (1995) z. B. wird die parasitäre Struktur nicht preisgegeben. Der Erzähler dieses Romans ist ein Video-Freak, der - in Parallele zum Roman "Grond" bzw. zu O. Wieners verbesserung von mitteleuropa, roman - "Roman" heißt: eine letzte Erinnerung an den traditionellen Roman. Dieser Roman wird "Romy" gerufen, in Parallele zu Grass' Fontane-Figur Fonty in Ein weites Feld und wohl auch in Parallele zu Romy Schneiders frühen Kitsch-Filmen, also in Konsequenz der Pulp-Anteile der Film-Kunst, der dieser "Roman" als Video-Freak huldigt. Menasse führt aber in den nur teilweise gekennzeichneten CakesZitaten nach Proust oder mit den (nicht gekennzeichneten) Zitaten aus Stifters Nachsommer bzw. aus der Letzten Mappe auch die traditionelle ideologiekritische Romantradition im Dilettanten-Video-Roman weiter: die Häuser im Waldviertler Ort Komprechts haben durch Anbauten für den Fremdenverkehr Hakenform; um welchen Fremdenverkehr, um welchen Steinbruch in Komprechts (Kompagnie rechts?) handelt es sich, der - im Jahr der Mauthausen-Gedenk-Veranstaltungen (1945-1995) - stillgelegt 
und zum Museum wird? Die parasitäre Strategie, nach der Menasse (z. B. im Politischen) schreibt, $m u \beta$ nicht (mit)gelesen werden. Die mögliche Tiefe unter der Oberfläche muß nicht bedacht werden: es wurde ein vielverkauftes und vielgelesenes Buch, das sich allen Mißverständnissen aussetzt, ohne Schaden zu nehmen.

So wie Oswald Wiener mit die verbesserung von mitteleuropa, roman 1969 ein epochales literaturtheoretisches Werk schrieb, so hat er das für die 90er Jahre mit dem hochliterarischen, von ihm aber als "Schundroman" bezeichneten Werk Nicht schon wieder. Eine auf einer Floppy gefundene Datei (1992) getan, das anonym erschien, mit einem Herausgeber Präkogler spielt (nach Philipp K. Dicks vor der Erkenntnis stehenden Precogs) und die hier nur angedeuteten Kippspiele der Gegenwartsliteratur und ihre scrotal-cerebralen Zusammenhänge so souverän-vielfältig wie floppy-banal zusammenfaßt. Nicht schon wieder..., das einen Geburtsbzw. Wiedererweckungsvorgang beschreibt, ist vermutlich ein hamletischer Protest-Titel und heißt: nicht schon wieder leben.

Martin Kubaczek hat das "Kippspiel von Fiction und Leserwirklichkeit", das mit dem Kippspielen in den Vermittlungsvorgängen der menschlichen Gattung korrespondiert, in einem Comic auf einer Kaugummi-Packung aufgefunden:
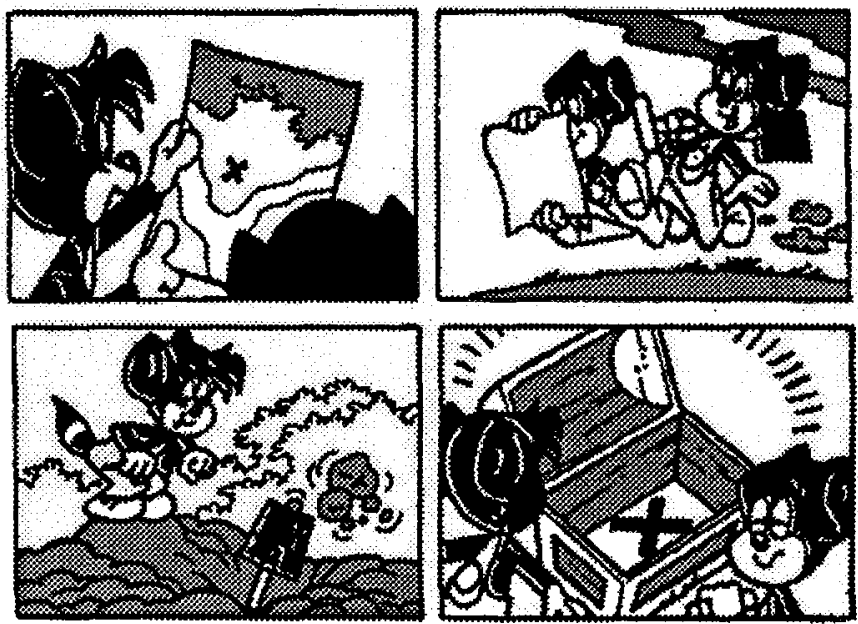
Der Baum der Erkenntnis, die Erfüllung der Wünsche, die Selbstverwirklichung durch Reichtum oder durchs Ungewöhnliche, die "Erlösung" aus unterschiedlichen - z. B. den grundsätzlichen geschlechtlichen Bedürfnissen etc., ist auf dem Comic in Form einer Schatzkarte und des durch sie gefundenen Schatzes gezeichnet. Es bleibt auch in dieser Geschichte beim Zeichen für die Wünsche. Immerhin liegt keine Bombe in der Schatzkiste. Das Zeichen hat jene X-Form, mit der auch Teile der Chromosomen-Sätze gezeichnet werden, die die Menschheit biologisch beschreiben. Es ist hier, im diesem Kaugummi-Comic, endlich das zur Vollendung fehlende X gefunden und das Spiel: Ich wünsch mir was, ich wünsch mir alles, Ich will überall sein, ich bin gar nicht da, Ich bin nicht Ich, ich bin ich, also der immanente Relationismus der Gattung, zu einem wie in Wirklichkeit enttäuschenden Ende geführt.

Ich habe einige gegenwärtige, teils bestsellerische, teils kanon-literarische Beispiele genannt und zeitgenössisch umrahmt. In der geschichts-immanenten Wirklichkeitsauffassung, wie die unsere seit der Aufklärung ist, werden alle Erscheinungen und ihr Verständnis im Verhältnis zu ihrer Zeit gesehen: die Zeit aber wird (demoskopisch) erhoben und die ErhebungsAbsicht ausverhandelt mit andern Absichten, d. h. bunt und widersprüchlich gemischt. Es kann, je nach Erhebungsmoment und Erheber, immer ebensogut anders sein. Das sind die Data. Es ist immer anders. Das sind die "Bedeutungen". Das Hohe ist die Erhebung des Banalen. 